\title{
The bosonic birthday paradox
}

\author{
Alex Arkhipov $1, *$ and Greg Kuperberg $2, \dagger$ \\ ${ }^{1}$ Massachusetts Institute of Technology \\ ${ }^{2}$ University of California, Davis
}

\begin{abstract}
We motivate and prove a version of the birthday paradox for $k$ identical bosons in $n$ possible modes. If the bosons are in the uniform mixed state, also called the maximally mixed quantum state, then we need $k \sim \sqrt{n}$ bosons to expect two in the same state, which is smaller by a factor of $\sqrt{2}$ than in the case of distinguishable objects (boltzmannons). While the core result is elementary, we generalize the hypothesis and strengthen the conclusion in several ways. One side result is that boltzmannons with a randomly chosen multinomial distribution have the same birthday statistics as bosons. This last result is interesting as a quantum proof of a classical probability theorem; we also give a classical proof.
\end{abstract}

The traditional birthday paradox says that given a calendar with $n$ days, there is a significant chance (bounded away from 0 ) that a room with $\Omega(\sqrt{n})$ people with uniformly random birthdays has two with the same birthday. Aaronson and Arkhipov [1] discuss the same paradox for randomly chosen bosons. Here we present a different treatment of the same problem. In fact we will present two "paradoxes". The first result (which Aaronson and Arkhipov derived, in a less general form) is that although bosons prefer to have the same birthday, they have the same asymptotic behavior in the birthday problem, up to constant factors, as distinguishable particles (boltzmannons). The second result is that they have exactly the same behavior, non-asymptotically, as $n$ i.i.d. boltzmannons whose common distribution is a randomly chosen point in the simplex of all distributions on $n$ configurations. This leads to an interesting result in classical probability with a quantum probability proof.

We assume that the Hilbert space for one particle is $\mathscr{H}=$ $\mathbb{C}^{n}$. We assume a self-adjoint birthday operator

$$
B: \mathscr{H} \rightarrow \mathscr{H}
$$

with eigenvalues $1,2, \ldots, n$ in some basis. The Hilbert space of $k$ bosons is then the symmetric power

$$
\left.S^{k}(\mathscr{H}) \cong \mathbb{C}\left(\begin{array}{l}
n \\
k
\end{array}\right)\right)
$$

using the multiset coefficient notation

$$
\left(\left(\begin{array}{l}
n \\
k
\end{array}\right)\right) \stackrel{\text { def }}{=}\left(\begin{array}{c}
n+k-1 \\
k
\end{array}\right) .
$$

In the terminology used for identical particles, the states of a basis of $\mathscr{H}$ are called modes.

In the traditional version of the classical birthday problem, we assume the uniform distribution on all $n^{k}$ choices of the birthdays of the $k$ people. The uniform distribution $\mu_{\text {unif }}(X)$ on any finite set $X$ can be characterized in either of two ways: It is the unique distribution with the most entropy, $\log |X|$; and the unique distribution with the most symmetry, $\operatorname{Sym}(X)$.

\footnotetext{
* Supported by an Akamai Foundation Fellowship.

$\dagger$ Partly supported by NSF grant CCF-1013079.
}

We will consider an analogue of the uniform distribution for a quantum system with a Hilbert space $\mathscr{H}$ : the mixed state $\rho_{\text {unif }}(\mathscr{H})$ whose density matrix is the scaled identity on $\mathscr{H}$. Like the classical state $\mu_{\text {unif }}$, the quantum state $\rho_{\text {unif }}(\mathscr{H})$ is the unique state on $\mathscr{H}$ with the most entropy, log $\operatorname{dim} \mathscr{H}$; and the unique state with the most symmetry, $U(\mathscr{H})$. Moreover, $\rho_{\text {unif }}(\mathscr{H})$ is the unique state that yields the distribution $\mu_{\text {unif }}(X)$ for any complete measurement that takes values in a set $X$.

We will use the uniform state $\rho_{\text {unif }}=\rho_{\text {unif }}\left(S^{k}(\mathscr{H})\right)$ on the joint Hilbert space of $k$ bosons. Then, the measurement $S^{k}(B)$ of all birthdays of $\rho_{\text {unif }}$ yields the uniform distribution $\mu_{\text {unif }}$ on configurations of $k$ unlabelled people with $n$ possible birthdays. (It is also standard to refer to unlabelled balls in labelled boxes, but we will stick to the birthday metaphor.) Moreover, this particular uniform state can be justified using less symmetry than the largest available unitary group $U\left(S^{k}(\mathscr{H})\right)$ :

Proposition 1. The state $\rho_{\text {unif }}$ on $S^{k}(\mathscr{H})$ is the unique state which is invariant under the unitary group $U(\mathscr{H})$.

Proof. Suppose that $\rho$ is a $U(\mathscr{H})$-invariant state on $S^{k}(\mathscr{H})$, i.e., a $U(\mathscr{H})$-invariant density operator. Schur's Lemma says that if $V$ is an irreducible complex representation of a group $G$, then every $G$-invariant operator on $V$ is proportional to the identity. Thus it is sufficient (and also necessary, if either $V$ is unitary or $G$ is compact) for $V$ to be irreducible. It is a standard fact of representation theory [4, $\S 6.1]$ that $S^{k}\left(\mathbb{C}^{n}\right)$ is an irreducible representation of $\operatorname{GL}(n, \mathbb{C})$. It is another standard fact [4, $\S 26.1]$ that $\operatorname{GL}(n, \mathbb{C})$ and $U(n)$ have the same irreducible representations, since the former is the complexification of the latter.

This symmetry implies that $\rho_{\text {unif }}$ is the $U(\mathscr{H})$-average of any state, since such an average must be invariant with respect to the action of $U(\mathscr{H})$.

Corollary 2. Putting $k$ bosons in any state $\sigma$ on $S^{k}(\mathscr{H})$, and then applying a Haar-random unitary matrix in $U(\mathscr{H})$ yields the state $\rho_{\text {unif. }}$

Aaronson and Arkhipov consider such an average for a particular choice of $\sigma$, where $\sigma$ is the pure state

$$
|\psi\rangle=|1,2,3, \ldots, k\rangle
$$


in which the $k$ bosons are in distinct modes (which requires $k \leq n)$. Another choice considered below is

$$
|\psi\rangle=|1,1,1, \ldots, 1\rangle
$$

in which the bosons are all in the same mode. There are many choices for $\sigma$, but Corollary 2 says that they all become the same when they are averaged.

We will now look at the asymptotics of $j$-fold birthdays in $\rho_{\text {unif. }}$. We will use the notation $f(n) \sim g(n)$ to mean that $f(n) / g(n) \rightarrow 1$, or equivalently that $f(n)=g(n)(1+o(1))$.

Theorem 3. Suppose that there are $k$ bosons with $n$ modes, suppose that they are in the uniform state $\rho_{\text {unif }}$, and suppose that $k \sim c n^{(j-1) / j}$ as $n \rightarrow \infty$, for some integer $j \geq 2$ and some constant $c>0$. Then the number of $j$-fold birthdays converges in distribution to a Poisson random variable with mean $c^{j}$, while the number of $(j+1)$-fold-or-more birthdays converges to 0 .

This is the same asymptotic answer as in the case of boltzmannons, except that the mean in that case is $c^{j} / j$ !. In fact, our argument in the case of bosons is very similar to a standard argument in the case of boltzmannons.

Proof. Recall that the joint measurement $S^{k}(B)$ of all of the birthdays yields the uniform distribution on $k$ unlabelled people among $n$ calendar days. The probability that the first birthday has at least $j+1$ people is

$$
\left(\left(\begin{array}{c}
n \\
k-j-1
\end{array}\right)\right) /\left(\left(\begin{array}{l}
n \\
k
\end{array}\right)\right) \sim \frac{k^{j+1}}{(n+k)^{j+1}}
$$

for fixed $j$ and $n, k \gg 1$. Taking $k=O\left(n^{(j-1) / j}\right)$ and summing over all $n$ days, the expected number of $(j+1)$-fold-or-more birthdays is $O\left(n^{-1 / j}\right)$, which vanishes as $n \rightarrow \infty$.

Meanwhile the probability that the first $\ell$ days each have at least $j$ people is

$$
\left(\left(\begin{array}{c}
n \\
k-j \ell
\end{array}\right)\right) /\left(\left(\begin{array}{l}
n \\
k
\end{array}\right)\right)=\prod_{a=0}^{j \ell-1} \frac{k-a}{n+k-a} \sim \frac{k^{j \ell}}{(n+k)^{-j \ell}},
$$

where the approximation holds for fixed $j$ and $\ell$ and $n, k \gg 1$. Summing over all $\left(\begin{array}{l}n \\ \ell\end{array}\right) \sim \frac{n^{\ell}}{\ell !}$ choices of the $\ell$ days, we obtain that if $X$ is a random variable representing the number of $j$ fold birthdays, then

$$
E\left[\left(\begin{array}{c}
X \\
\ell
\end{array}\right)\right] \sim \frac{c^{j \ell}}{\ell !}
$$

So in the limit, the $\ell$ th factorial moment is $c^{j \ell}$, which the same answer in the limit as a Poisson random variable with mean $c^{j}$. To conclude the argument, the Poisson distribution is determined by its moments.

The calculation for the narrow question of the probability of at least one repeated birthday is simpler. The probability that all of the birthdays are distinct is

$$
\left(\begin{array}{l}
n \\
k
\end{array}\right) /\left(\left(\begin{array}{l}
n \\
k
\end{array}\right)\right)=\prod_{a=0}^{k-1} \frac{1-\frac{a}{n}}{1+\frac{a}{n}} \sim e^{-k^{2} / n}
$$

as long as $k=o\left(n^{3 / 4}\right)$. The approximation is established by taking the logarithm of both sides and then applying the Taylor series estimate

$$
\ln \frac{1-x}{1+x}=-2 x+O\left(x^{3}\right) .
$$

Corollary 4. For $n$ modes, we need $k \sim \sqrt{n \ln 2}$ bosons to expect a repeated birthday with majority probability.

This differs by only a constant factor from the $k \sim \sqrt{2 n \ln 2}$ people needed to expect a repeated birthday in the classical birthday problem with distinguishable people.

Remark. We should say something about independent but non-uniform bosons. The notion of independence for bosons is subtle. One reasonable and widely used notion is to first choose a distribution $\mu$ for the birthdays of one boson, and to model it by a diagonal density matrix in the birthday basis. Then there is a unique distribution on $k$ bosons such that if $k-1$ of the bosons are fixed, the conditional distribution of the last one is given by $\mu$. This distribution is also a thermal state, also known as a Maxwell-Gibbs state, for noninteracting bosons. It was discovered by Bose and Einstein that under fairly mild assumptions on $\mu$, almost all of the bosons have the most likely birthday. This paradox is commonly known as Bose-Einstein condensation.

Corollary 2 implies an interesting second model for the joint distribution of birthdays of $k$ bosons.

Theorem 5. The joint birthday distribution of $k$ bosons in the uniform state $\rho_{\text {unif }}$ is identical to the average of $k$ i.i.d. boltzmannons, if their common distribution is given by a uniformly random point in the simplex of distributions on the $n$ birthdays.

By combining with the induced uniform distribution on the birthday measurement, we obtain a corollary of Theorem 5 that equates two distributions in classical probability.

Corollary 6. Consider a town in which all families first agree to have children according to a common distribution on the days of the year, which itself is chosen uniformly from the simplex of all distributions. Then the children's birthdays behave as if the children were unlabelled, i.e., if we make a table that only gives the number of children born on each day, then all such tables are equally likely.

In other words, the uniform average of all multinomial distributions on multisubsets of size $k$ in a set of size $n$, is the uniform distribution on multisubsets.

Proof of Theorem [5. Recall that the Hilbert space of $k$ boltzmannons is $\mathscr{H}^{\otimes k}$. Consider the state $\sigma=(|\psi\rangle\langle\psi|)^{\otimes k}$, first for some fixed choice of $|\psi\rangle \in \mathscr{H}$. This $\sigma$ yields independently distributed birthdays for the $k$ boltzmannons, and the distribution of each one is given by the measurement of one copy of $|\psi\rangle$. Meanwhile, $\sigma$ is evidently a pure symmetric state, which means that these boltzmannons are also bosons. By Corollary 2 the average of all choices of $\sigma$, with respect to Haar measure on $U(\mathscr{H})$, is the bosonic state $\rho_{\text {unif. }}$. 
The Haar distribution of $|\psi\rangle$, or equivalently one column of a matrix in $U(n)$, is given by Haar measure on the manifold of pure states $\mathbb{C P}^{n-1}$. The induced distribution of the birthday measurement is given by the moment map

$$
m: \mathbb{C P}^{n-1} \rightarrow \Delta_{n-1}
$$

to the simplex of distributions on $n$ configurations [2, §6.4]. This moment map preserves normalized measure [2, §6.6]. Thus a random choice of $\sigma$ amounts to a random distribution on each birthday, drawn uniformly from the simplex of distributions. This establishes the claim of the theorem.

Theorem 5yields a quantum proof of a classical probability result, Corollary 6. We also obtained a classical proof of the same result.

Classical proof of Corollary 6 . The argument uses a variation of the stars-and-bars notation for multisets [3] that is also used to prove the identity (1). Namely, we write a star for each of the $k$ children, with $n-1$ separating bars between the $n$ calendar days. For example, if there are $k=4$ children and $n=$ 6 birthdays, then one possible choice for all of the birthdays is

$$
\star \star|\star| \star \star \|,
$$

in which two children are born on the first day, one on the second day, one on the fourth day, and none on the other days. We first choose locations of $n-1$ bars independently and uniformly on the unit interval $I=[0,1]$. This separates the interval into $n$ subintervals of length

$$
p_{1}+p_{2}+\ldots+p_{n}=1
$$

and we claim that the lengths of these subintervals are given by a uniformly random point in the simplex of distributions.
(Because, if we first take the bars to be numbered, they are distributed according to uniform measure on $[0,1]^{n-1}$. Then, erasing the numbers yields the quotient $[0,1]^{n-1} / S_{n-1}$, which is a simplex and also has uniform measure. Then, taking the differences of successive points to obtain the probabilities $p_{j}$ is a linear isomorphism, which also preserves uniform measure.) Then, if each child's birth is represented by a star which is also at a uniformly random position in $[0,1]$, the probability of the $j$ th birthday is exactly $p_{j}$, the length of the $j$ th interval.

We note that the ordering of the stars and bars determines the number of children with each birthday. We claim that these multiset choices are all equally likely, as if the children had been bosons (with no distinguishing state other than the date of birth). This is made clear if we equivalently choose $n-$ $1+k$ points independently from $I$ all at once, and then choose a random subset of $n-1$ points to be the bars and the other $k$ points to be the stars. These $\left(\left(\begin{array}{l}n \\ k\end{array}\right)\right)=\left(\begin{array}{c}n-1+k \\ k\end{array}\right)$ equally likely choices exactly correspond to a multiset choice of $k$ unlabelled children distributed among $n$ days, as claimed.

We conclude with a version of the birthday paradox for fermions.

Theorem 7 (Pauli). Given $k$ fermions in any state on the exterior power $\Lambda^{k}(\mathscr{H})$, there is no chance that any two have the same birthday.

We leave the question of an anyonic birthday paradox, including non-abelian anyons, as a topic for future work.

\section{ACKNOWLEDGMENTS}

The authors would like to thank Scott Aaronson for suggesting the problem.
[1] Scott Aaronson and Alex Arkhipov, The computational complexity of linear optics, arXiv:1011.3245.

[2] Ana Cannas da Silva, Symplectic geometry, Handbook of differential geometry. Vol. II, Elsevier/North-Holland, 2006, arXiv:math/0505366, pp. 79-188.

[3] William Feller, An introduction to probability theory and its applications. Vol. I, 3rd ed., John Wiley \& Sons Inc., 1968.
[4] William Fulton and Joseph Harris, Representation theory, third ed., Graduate Texts in Mathematics, vol. 129, Springer-Verlag, New York, 1998. 